\title{
Scientific Note/Comunicacão Cientifica Primeiro Registro de Aricoris campestris (H. Bates) (Lepidoptera: Riodinidae) em Palma Forrageira Nopalea cochenillifera (L.) Salm-Dyck (Cactaceae) no Brasil
}

\author{
Mileny dos Santos de Souza ${ }^{\circledR}$, José Thyago Aires Souza1, Guilherme Ferreira Costa Lima², \\ Márcio Raimundo de Medeiros², Robério de Oliveira ${ }^{1}$ \& Jacinto de Luna Batista ${ }^{1}$
}

1. Programa de Pós-Graduação em Agronomia, Centro de Ciências Agrárias, Universidade Federal da Paraíba, CCA/UFPB. 2. Empresa de Pesquisa Agropecuária do Rio Grande do Norte, EMPARN.

\section{EntomoBrasilis 11 (2): $142-143$ (2018)}

Resumo. A palma Nopalea cochenillifera (L.) foi introduzida no Brasil para produção do corante carmim, porém logo se descobriu seu potencial como forrageira. Até então, os relatos eram de que essa planta tinha como principais insetos-praga um grupo de cochonilhas. Este trabalho relata pela primeira vez a presença de Aricoris campestris (H. Bates) infestando essa cultura.

Palavras-chave: Cactáceas; forrageiras; insetos-praga; lepidópteros; palma doce.

\section{First Record of Aricoris campestris (H. Bates) (Lepidoptera: Riodinidae) in Cacti Nopalea cochenillifera (L.) Salm-Dyck (Cactaceae) in Brazil}

Abstract. The Nopalea cochenillifera (L.) cacti was introduced in Brazil to produce the carmine dye, but soon its potential as forage was discovered. Until then reports were that this plant had as main insects-pest a group of mealybugs. This work reports for the first time the presence of Aricoris campestris (H. Bates) infesting this culture.

Keywords: Cacti; forage; lepidopteran; pest; sweet cacti.

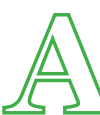
palma-forrageira, Nopalea cochenillifera (L.) SalmDyck, é uma cactácea de origem mexicana, adaptada e amplamente cultivada no Semiárido nordestino (NoBEL 2001; Oliveira et al. 2011). De significativo valor nutricional, é usada para alimentar o rebanho de bovinos e caprinos nos períodos de estiagem, por tratar-se também de uma fonte de água para os animais (SILVA \& SAMPAIO 2015).

As cactáceas forrageiras cultivadas no Nordeste brasileiro têm sido atacadas por um lepidóptero que, com pouco tempo de instalado no plantio, multiplica-se rapidamente, podendo atingir o nível de praga. No presente trabalho, lagartas de lepidópteros foram coletadas diretamente nas raquetes de $N$. cochenillifera, em março de 2014, nos municípios de São Vicente do Seridó, PB (6 $6^{\circ} 56^{\prime} 2^{\prime \prime} \mathrm{S}, 36^{\circ} 24^{\prime} 7^{\prime \prime} \mathrm{W}$ ) e Zabelé, PB ( $\left.8^{\circ} 4^{\prime} 33^{\prime \prime} \mathrm{S}, 37^{\circ} 5^{\prime} 52^{\prime \prime} \mathrm{W}\right)$, e em outubro de 2016 em Cruzeta, RN ( $6^{\circ} 24^{\prime} 43^{\prime \prime}$ S, 36 $36^{\circ} 47^{\prime} 24^{\prime \prime}$ W) (Figura 1). As lagartas foram coletadas com o auxílio de uma pinça, acondicionadas em recipientes plásticos transparentes, com pequenos furos na parte superior, e transportadas até o Laboratório de Entomologia do Departamento de Fitotecnia e Ciências Ambientais do Centro de Ciências Agrárias da Universidade Federal da Paraíba, CCA/UFPB, município de
Areia, PB. Em laboratório, as lagartas foram alimentadas com brotos de $N$. cochenillifera e mantidas em câmara climatizada, em temperatura de $27 \pm 1^{\circ} \mathrm{C}$ e umidade de $70 \pm 10 \%$. Ao atingirem a fase pupal, foram transferidas para uma gaiola confeccionada em arame galvanizado e coberta com tecido voil, enquanto aguardou-se a emergência dos adultos.

A identificação foi realizada com o auxílio de um microscópio estereoscópico, a partir da observação de espécimes adultos, com o uso da chave de classificação especifica para lepidópteros de Costa Lima (1950), alcançando a família Riodinidae, confirmando a espécie Aricoris campestris (H. Bates) (Lepidoptera: Riodinidae), com uso de literatura especializada (ButTERFlies of AMERicA Foundation 2009). Observou-se junto às pupas de $A$. campestris a emergência de parasitoides da família Chalcididae, sendo esse identificado em nível de família, por meio da chave para classificação de himenópteros de Costa Lima (1962), em 30 pupas do hospedeiro observou-se a emergência de oito parasitoides.

As lagartas de $A$. campestris se alimentam principalmente das brotações da palma, raspando-as em seus primeiros estádios,
Edited by:

Rodrigo Souza Santos

Article History:

Received: o6.vii.2017

Accepted: 30.x.2010
Corresponding author:

Mileny dos Santos de Souza

\} mileny.lopes67@gmail.com

(7) http://orcid.org/0000-0003-0721-6800
Funding agencies:

$\Delta$ Without funding declared 


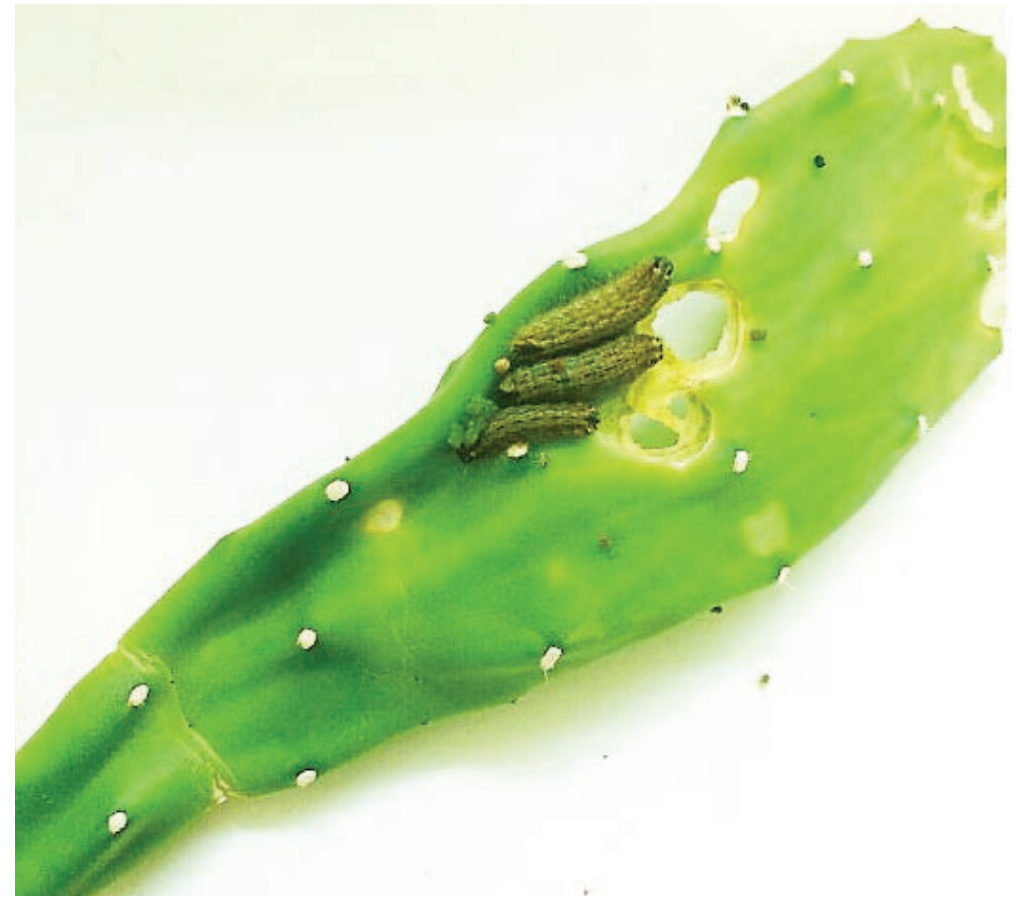

Figura 1. Raquete da palma-forrageira Nopalea cochenillifera infestada por lagartas de Aricoris campestris coletadas no município de Cruzeta, RN. (Foto: Souza, M.S).

causando posterior necrose da área, e quando essas alcançam um tamanho maior, perfuram as raquetes. $\mathrm{O}$ dano pode ser elevado, provocando a perda total da raquete.

A espécie $A$. campestris já foi relatada anteriormente em pesquisas na Região Nordeste, em Tecoma stans (L.) Kunth (Bignoniaceae), sendo constatado o estágio adulto nas estruturas florais em busca de pólen e néctar, no entanto, sem causar danos às plantas (DiAs 2015). Esse lepidóptero tem importantes interações simbióticas com formigas do gênero Solenopsis (Hymenoptera: Formicidae) (Kaminski \& CARVAlHo-FilHo 2012).

\section{REFERÊNCIAS}

Butterflies of America Foundation, 2009. Família Riodinidae Grote, 1895 (1827). Disponível em: http://www. butterfliesofamerica.com/L/Riodinidae.htm. [Acesso em: 31.vi.2017].

Costa Lima, A.M. da, 1950. Insetos do Brasil. Lepidópteros, $2^{\text {a }}$ parte, p.1-10o. In: Insetos do Brasil: Lepidópteros. Rio de Janeiro: Escola Nacional de Agronomia (Série Didática, 8). $420 \mathrm{p}$.

Costa Lima, A.M. da, 1962. Insetos do Brasil. Hymenópteros, $2^{\text {a }}$ parte, p.173-194. In: Insetos do Brasil: Himenópteros. Rio de Janeiro: Escola Nacional de Agronomia (Série Didática, 14). $393 \mathrm{p}$.
Dias, M.A.B., 2015. Estratégias comportamentais de visitantes florais lepidópteros e himenópteros em Tecoma stans (L.) Kunth (Bignoniaceae) diante da sazonalidade. Trabalho de Conclusão de Curso (Ciências Biológicas) - Universidade Federal de Campina Grande, Centro de Saúde e Tecnologia Rural. 58 f.

Kaminski,L.A.\&F.S.Carvalho-Filho, 2012. Lifehistory of Aricoris propitia (Lepidoptera: Riodinidae) - a myrmecophilous butterfly obligately associated with fire ants. Psyche, 2012: 1-10. DOI: https://doi.org/10.1155/2012/126876.

Nobel, P.S., 2001. Biologia ambiental, p. 36-48. In: Barbera, G., P. Iglese \& E. Pimienta-Barrios (Orgs.). Agroecologia, cultivo e usos da palma forrageira. Paraíba: SEBRAE/PB. 216 p.

Oliveira, A.S.C., F.N. Cavalcante Filho, A.H.N. Rangel \& K.B.P. Lopes, 2011. A palma forrageira: alternativa para o Semiárido. Revista Verde de Agroecologia e Desenvolvimento Sustentável, 6: 49-58. DOI: https://doi.org/10.18378/rvads. v12i1.4890.

Silva, R.R. \& E.V.S.B. Sampaio, 2015. Palmas forrageiras Opuntia ficus-indica e Nopalea cochenillifera: sistemas de produção e usos. Revista Geama, 1: 131-141.

$$
* * * * * * * * * *
$$

\section{Suggestion citation:}

Souza, M.S., J.T.A. Souza, G.F.C. Lima, M.R. Medeiros, R. Oliveira \& J.L. Batista, 2018. Primeiro Registro de Aricoris campestris (H. Bates) (Lepidoptera: Riodinidae) em Palma Forrageira Nopalea cochenillifera (L.) Salm-Dyck (Cactaceae) no Brasil. EntomoBrasilis, 11 (2): $142-143$. Available on: doi:10.12741/ebrasilis.v11i2.725
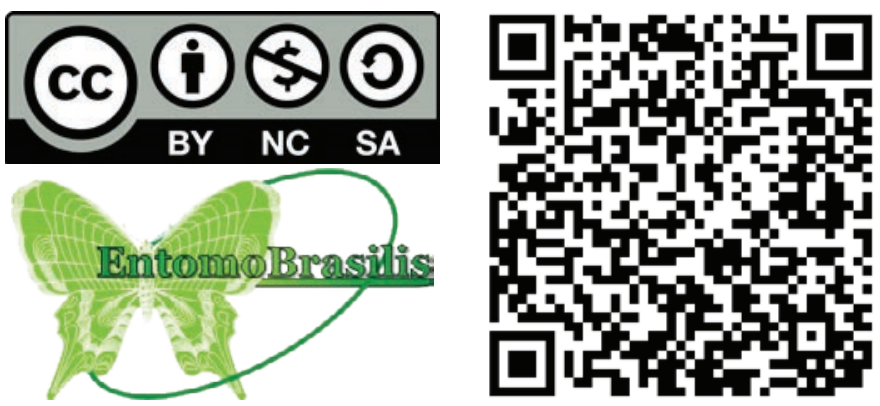